\title{
MatchingTools: a Python library for symbolic effective field theory calculations
}

\author{
Juan C. Criado ${ }^{\mathrm{a}, *}$ \\ ${ }^{a}$ CAFPE and Departamento de Física Teórica y del Cosmos, Universidad de Granada, E-18071 Granada, Spain
}

\begin{abstract}
MatchingTools is a Python library for doing symbolic calculations in effective field theory. It provides the tools to construct general models by defining their field content and their interaction Lagrangian. Once a model is given, the heavy particles can be integrated 'out at the tree level to obtain an effective Lagrangian in which only the light particles appear. After integration, some of the terms of the resulting Lagrangian might not be independent. MatchingTools contains functions for transforming these terms to rewrite them in terms of any chosen set of operators.
\end{abstract}

Keywords: effective; tree; integration; matching; redundancies; python;

\section{PROGRAM SUMMARY}

Program Title: MatchingTools

Licensing provisions: MIT

Programming language: Python (compatible with versions 2 and 3)

"Nature of problem:

The program does two kinds of calculations: computing an effective

Lagrangian for the light fields of a field theory by integrating out at the tree level the heavy fields and performing algebraic manipulations with tensors in the (effective) Lagrangian.

Solution method:

The tree level integration of heavy fields is done by substituting them inside the Lagrangian by a covariant derivative expansion of the solution to their equations of motion. The transformation of Lagrangians is implemented as an algorithm for finding patterns of tensor products and replacing them by sums of other products.

\section{Introduction}

When studying physical phenomena in the framework of field theory, it is often convenient to describe the low energy behavior of the system in a way that does not involve the heavy degrees of freedom. A low energy effective theory can be derived from a more fundamental one, when the latter is known. The connection between both descriptions is done by integrating out the heavy fields. The basic idea is to find the set the effective interactions of the light fields such that the corresponding low energy observables match, to the desired precision, those computed using the full theory [1, 2].

An important example arises in particle physics, when studying extensions of the Standard Model. The latest experimental results from the Large Hadron Collider (LHC) do not show any evidence of direct production of new particles (see for example [3, 4, 5, 6]). Therefore, the discovery of new physics arising

\footnotetext{
${ }^{*}$ Corresponding author.
}

E-mail address: jccriadoalamo@ugr.es within the range of energies of the current phase of the LHC seems more and more unlikely.

In view of this perspective, it is interesting to extract features of physics at higher, currently unreachable energies by using precision measurements. This can be done using an effective theory approach. In the Standard Model Effective Field Theory (SMEFT), the Standard Model is extended to include non-renormalizable operators (see [7] and references therein). In this setting, the high energy physics is parametrized in low energy by the coefficients of the new operators. These coefficients can be constrained by the experimental data $[8,9,10,11$, $12,13,14,15,16,17$. Another effective approach to extending the Standard Model is the Higgs effective theory, in which the gauge symmetry is realized non-linearly [18, 19, 20, 21].

Many of the proposed theories for physics beyond the Standard Model predict the existence of new, heavy particles [22, $23,24,25]$ The result of integrating out these heavy fields is the collection of the corresponding coefficients of the operators of the SMEFT [26, 27, 28, 29].

The procedure of matching can be described algebraically in terms of tensor calculus manipulations involving the computation of functional derivatives and the substitution of heavy fields by other previously obtained expressions $[30,31,32,33,34,35$, $36,37,38,39,40,41]$. The complexity of the process quickly grows with the number of heavy fields and their interactions. It is in this context where the development of a computer tool to automatize the process becomes necessary.

MatchingTools can perform tree-level integration of heavy fields in any given Lagrangian. It has been developed with the application to the SMEFT in mind, but it is able to work with any situation describable by a Lorentz invariant field theory in which the high energy degrees of freedom to be removed are scalars, vector-like or Majorana fermions, or vectors. By introducing the generic solution to their equations of motion, other types of fields can be treated as well. The validity of MatchingTools extends to any level in the expansion in inverse powers of the cut-off energy of the effective theory. 
The Lagrangian resulting from integration usually contains redundancies: operators that can be written in terms of others using identities of the symmetry group, integration by parts or equations of motion of the light fields [42, 43, 44, 45]. A complete set of operators that are independent under this set of transformations is called a basis. Several such bases have been described [46, 47, 48].

The transformation of the results of an integration to a chosen basis can also be done using MatchingTools. One should introduce the identities between tensor expressions needed to transform some operators into others, as well as the desired basis.

There are other tools for the manipulation of bases of operators, such as Rosetta [49]. The portion of MatchingTools that deals with this calculations differs from it in two main points: first, it allows not only for the transformations between sets of already independent operators, but for the transformation of any set of operators into a basis. Moreover, MatchingTools has the ability of doing transformations not with the operators themselves, but with parts of them, allowing for general transformations between parts of tensor expressions into others. Actually, MatchingTools can be used as system for tensor calculus manipulations, not necessarily in the context of an effective field theory. It provides a fast way of doing complex symbolic calculations with many fields and terms involved, which is safe against algebraic errors.

A direct application of MatchingTools, which has also served as an extensive check of its validity, is the integration of all possible new fields that have linear gauge-invariant renormalizable couplings to the Standard Model, keeping terms up to dimension six in the results [50].

A package that implements a similar way of dealing with the specification of models is FeynRules [51, 52], thought its objectives are completely distinct. One possible direction for future work with MatchingTools is making the connection with FeynRules.

Among other computer tools for calculations in the context of the SMEFT we have DsixTools [53] (which allows for several calculations including a case of tree level matching) and SMEFTsim [54] (which is able to produce theoretical predictions and constraints for the Wilson coefficients of the dimension 6 SMEFT).

MatchingTools is available in GitHub (https://github.com/jccriado/matchingtools) and in the PyPI repository (https://pypi.python.org/pypi/matchingtools/), so it can be installed using pip [55] as

\section{pip install matchingtools}

This article is organized as follows: section 2 describes the procedure and the formulas that the library uses for the integration of heavy particles. Sections 3, 4, 5 and 6 explain the features of MatchingTools and how to use it. Section 7 proposes a simple example that serves to see the library in action and as a test case. Some extra features for the applications in physics beyond the Standard Model are introduced in section 8 . Section 9 is an explanation of how to integrate out new types of fields that are not included in MatchingTools.

\section{Theoretical framework}

\subsection{Tree level integration}

Starting with a high energy theory with action $S[\phi, \Phi]$ depending on the light and heavy fields $\phi$ and $\Phi$ the effective action $S_{\text {eff }}$ for the light fields is obtained through:

$$
e^{i S_{e f f}[\phi]} \propto \int \mathcal{D} \Phi e^{i S[\phi, \Phi]}
$$

where $\int \mathcal{D} \Phi$ means integrating over all the configurations of the heavy fields $\Phi$. The configuration that contributes the most to this integral is the classical configuration $\Phi_{c}$, which extremizes the action. To leading order in $\left(\Phi-\Phi_{c}\right)$, we get

$$
S_{e f f}[\phi]=S\left[\phi, \Phi_{c}\right],
$$

known as the tree level approximation. It is the one that we will use in this article.

\subsection{Equations of motion and their solution}

To obtain the classical configuration of the heavy fields it is necessary to solve their equations of motion. They are determined from the condition: $\delta S / \delta \Phi=0$.

The variation of a local action $S=\int d^{m} x \mathcal{L}$ can be written as

$$
\frac{\delta S}{\delta \Phi}=\sum_{n}(-1)^{n} D_{\mu_{1}} D_{\mu_{2}} \cdots D_{\mu_{n}} \frac{\partial \mathcal{L}}{\partial\left(D_{\mu_{n}} \cdots D_{\mu_{2}} D_{\mu_{1}} \Phi\right)},
$$

which we have expressed in terms of a covariant derivative $D$ for the gauge group of the low energy effective field theory. It is convenient to split the action into a quadratic and an interaction part:

$$
S=\int d^{m} x \mathcal{L}_{\text {quad }}+S_{\text {int }}, \quad \mathcal{L}_{\text {quad }}=-\Phi^{\dagger} P \Phi,
$$

where $P$ is some differential operator.

For the bosonic fields, the solution to the equation of motion will be given by the application of the inverse of $P$ to a functional derivative of the interaction action. $P^{-1}$ can be expanded in each case in powers of $D_{\mu} / M$. For fermions, the solution will be given as a system of two equations. Recursive substitution of one into the other will give the solution to any order in $D_{\mu} / M$. Because we usually limit the dimension of the operators appearing in the effective Lagrangian we will only need to substitute a finite number of terms of these infinite expansions.

Several fields can be integrated out together. The solution to the equation of motion of each of them may involve the others, but they can be replaced recursively by their corresponding solutions to the equations motion to obtain solutions that only involve the light fields to the desired order.

The Lagrangian $\mathcal{L}_{\text {quad }}$ and the solution to the equations of motion is, for the following types of fields:

- Real scalar:

$$
\begin{aligned}
\mathcal{L}_{\text {quad }} & =-\frac{1}{2} \Phi\left(D^{2}+M^{2}\right) \Phi, \\
\Phi_{c} & =\sum_{n=0}^{\infty}(-1)^{n} \frac{D^{2 n}}{M^{2 n+2}} \frac{\delta S_{\text {int }}}{\delta \Phi} .
\end{aligned}
$$


- Complex scalar:

$$
\begin{aligned}
\mathcal{L}_{\text {quad }} & =-\Phi^{\dagger}\left(D^{2}+M^{2}\right) \Phi, \\
\Phi_{c} & =\sum_{n=0}^{\infty}(-1)^{n} \frac{D^{2 n}}{M^{2 n+2}} \frac{\delta S_{i n t}}{\delta \Phi^{\dagger}}, \\
\Phi_{c}^{\dagger} & =\sum_{n=0}^{\infty}(-1)^{n} \frac{D^{2 n}}{M^{2 n+2}} \frac{\delta S_{i n t}}{\delta \Phi} .
\end{aligned}
$$

- Real vector:

$$
\begin{aligned}
\mathcal{L}_{\text {quad }} & =\frac{1}{2} V^{\mu}\left\{\eta_{\mu v}\left(D^{2}+M^{2}\right)-D_{v} D_{\mu}\right\} V^{v}, \\
V_{c} & =-\frac{1}{M^{2}} \sum_{n=0}^{\infty} Q^{n} \frac{\delta S_{\text {int }}}{\delta V},
\end{aligned}
$$

where $Q$ is a differential operator that acts on a Lorentz vector and gives a Lorentz vector as:

$$
(Q V)_{\mu}:=\frac{D_{v} D_{\mu}-\eta_{\mu \nu} D^{2}}{M^{2}} V^{v}
$$

- Complex vector:

$$
\begin{aligned}
\mathcal{L}_{\text {quad }} & =V^{\dagger \mu}\left\{\eta_{\mu \nu}\left(D^{2}+M^{2}\right)-D_{v} D_{\mu}\right\} V^{v}, \\
V_{c} & =-\frac{1}{M^{2}} \sum_{n=0}^{\infty} Q^{n} \frac{\delta S_{i n t}}{\delta V^{\dagger}}, \\
V_{c}^{\dagger} & =-\frac{1}{M^{2}} \sum_{n=0}^{\infty} Q^{n} \frac{\delta S_{i n t}}{\delta V} .
\end{aligned}
$$

- Vector-like fermion (using two-component spinor notation):

$$
\begin{aligned}
\mathcal{L}_{\text {quad }}= & i F_{L \dot{\alpha}}^{\dagger} \bar{\sigma}_{\mu}^{\dot{\alpha} \alpha} D^{\mu} F_{L \alpha}+i F_{R}^{\dagger \alpha} \sigma_{\alpha \dot{\alpha}}^{\mu} D_{\mu} F_{R}^{\dot{\alpha}} \\
& -M\left(F_{L \dot{\alpha}}^{\dagger} F_{R}^{\dot{\alpha}}+F_{R}^{\dagger \alpha} F_{L \alpha}\right), \\
\left(F_{c}\right)_{L \alpha}= & \frac{i}{M} \sigma_{\alpha \dot{\alpha}}^{\mu} D_{\mu} F_{R}^{\dot{\alpha}}+\frac{1}{M} \frac{\delta S_{i n t}}{\delta F_{R}^{\dagger \alpha}} \\
\left(F_{c}\right)_{R}^{\dot{\alpha}}= & \frac{i}{M} \bar{\sigma}_{\mu}^{\dot{\alpha} \alpha} D^{\mu} F_{L \alpha}+\frac{1}{M} \frac{\delta S_{i n t}}{\delta F_{L \dot{\alpha}}^{\dagger}}, \\
\left(F_{c}\right)_{L \dot{\alpha}}^{\dagger}= & -\frac{i}{M} \sigma_{\alpha \dot{\alpha}}^{\mu} D^{\mu} F_{R}^{\dagger \alpha}-\frac{1}{M} \frac{\delta S_{i n t}}{\delta F_{R}^{\dot{\alpha}}}, \\
\left(F_{c}\right)_{R}^{\dagger \alpha}= & -\frac{i}{M} \bar{\sigma}_{\mu}^{\dot{\alpha} \alpha} D^{\mu} F_{L \dot{\alpha}}^{\dagger}-\frac{1}{M} \frac{\delta S_{i n t}}{\delta F_{L \alpha}} .
\end{aligned}
$$

- Majorana fermion:

$$
\begin{aligned}
\mathcal{L}_{\text {quad }}= & i F_{\dot{\alpha}}^{\dagger} \bar{\sigma}_{\mu}^{\dot{\alpha} \alpha} D^{\mu} F_{\alpha} \\
& -\frac{1}{2}\left(\epsilon^{\alpha \beta} F_{\beta} F_{\alpha}+F_{\dot{\alpha}}^{\dagger} \epsilon^{\dot{\alpha} \dot{\beta}} F_{\dot{\beta}}^{\dagger}\right), \\
\left(F_{c}\right)_{\beta}= & \epsilon_{\alpha \beta}\left(i \bar{\sigma}_{\mu}^{\dot{\alpha} \alpha} D^{\mu} F_{\dot{\alpha}}^{\dagger}+\frac{\delta S_{i n t}}{\delta F_{\alpha}}\right), \\
\left(F_{c}\right)_{\dot{\beta}}^{\dagger}= & \epsilon_{\dot{\beta} \dot{\alpha}}\left(i \bar{\sigma}_{\mu}^{\dot{\alpha} \alpha} D^{\mu} F_{\alpha}+\frac{\delta S_{i n t}}{\delta F_{\dot{\alpha}}^{\dagger}}\right) .
\end{aligned}
$$

\section{Creation of models}

In this section we will describe how to create a model using the module matchingtools. core. It assumes that the classes and functions that are used are in the namespace. To import all the classes and functions that appear here do

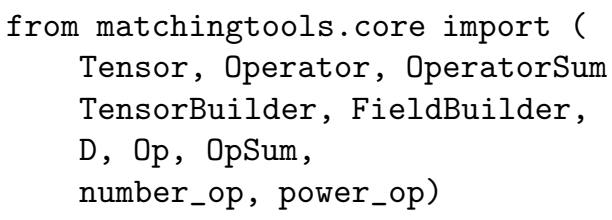

The from ... import ... style is recommended, as the expressions that appear when using this library tend to be long, so having the short names directly accessible is preferable.

\subsection{Creation of tensors and fields}

In MatchingTools, the basic building blocks for everything are the objects of the class Tensor, which we simply call tensors here. Examples of tensors are fields (light and heavy), symmetry group related tensors (such as Pauli matrices) or coupling constants (including gauge couplings, Yukawa couplings and masses).

Tensors have an attribute is_field that is True if and only if they are spacetime dependent (i.e., they are fields). Fields can have derivatives applied to them. The attribute num_of_der counts the number of derivatives that apply to a field. Derivatives are understood here to be covariant derivatives $D_{\mu}$ corresponding to the gauge group of the low energy effective theory. Each derivative applies only to one field. The Leibniz rule is used whenever a derivative of a product is encountered. Tensors can be either commuting of anti-commuting, which is distinguished by the attribute statistics. It can be set equal to either boson or fermion, both being variables defined in this module. Finally, all tensors have an attribute indices, a list of integer numbers representing their tensor indices; and an attribute name, an identifier. Other attributes, content and exponent, are for internal use. Names starting with the character ' $\$$ ' are also reserved for internal calculations.

To create the tensors and fields of a model, the classes TensorBuilder and FieldBuilder should be used. For example, the Pauli matrices $\sigma_{i j}^{a}$ could be defined as

$$
\text { sigma }=\text { TensorBuilder ("sigma") }
$$

and then used when needed as sigma (i1, i2, i3) where i1, $i 2$ and $i 3$ are the indices. Similarly, a boson field $\phi$ (with its conjugate $\phi^{*}$ ) and a fermion $f$ (with its separate chiralities and their conjugates) are defined as

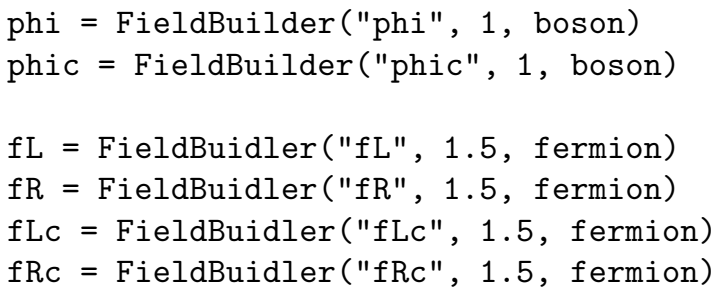

The second argument of FieldBuilder is the dimension of the field. 


\subsection{Definition of the interaction Lagrangian}

Once all the tensors are created, we are ready to define the interaction Lagrangian. It should be a sum of operators, which in turn are just products of fields. Using the functions OpSum and $0 \mathrm{p}$ :

$$
\text { int_lag }=-0 p \operatorname{Sum}(0 \mathrm{p}(\ldots), \mathrm{Op}(\ldots), \ldots)
$$

The minus sign is defined for operator sums and individual operators. The function OpSum creates an object of the class OperatorSum, a container for a list of operators representing their sum. The function $0 p$ creates an Operator that contains a list of tensors and represents their product:

$$
\begin{aligned}
& \text { Op (tensor } 1(i 1, i 2, \ldots), \\
& \quad \operatorname{tensor} 2(i 3, i 4, \ldots), \ldots)
\end{aligned}
$$

Positive indices are used to express contraction. During the creation of the model, any index should be contracted with another, so we will only use here positive ones. When indices are repeated inside the same operator, the corresponding contraction is understood. For example, the product of tensors $r_{i j} s_{\text {limnm }} t_{n j l}$ would be written as

$$
O p(r(0,1), s(3,0,4,5,4), t(5,1,3))
$$

To introduce a covariant derivative inside an operator, the appropriate function is D, whose first argument is the Lorentz index of the derivative and whose second one is the tensor to which it is to be applied:

$$
D(i 1, \text { tensor }(i 2, \ldots))
$$

For numeric coefficients, the function number_op creates an operator with only one special tensor representing a number (its name is "\$number" and has an attribute content with the actual number). Multiplication is defined for operators, so the operator $i V_{\mu} S_{a}^{*} D_{\mu} S_{a}$ can be expressed as

$$
\text { number_op }(1 j) * 0 p(V(0), S c(1), D(0, S(1)))
$$

Tensors representing a symbolic constant exponentiated to some power can be created using the function power_op, that takes the base (a string) and the exponent (a number) (represented by an extra internal attribute of tensors: exponent) and optionally some indices and returns an operator containing only the corresponding tensor. This is useful specially for the masses of the heavy particles, which tend to appear several times with different powers in all calculations.

A summary of the tools presented in this section is shown in table 1

\subsection{Dealing with spinors}

MatchingTools uses the two-component spinor formalism to treat spinor fields following the conventions in [56]. The module matchingtools.core defines the following tensors to work with them:

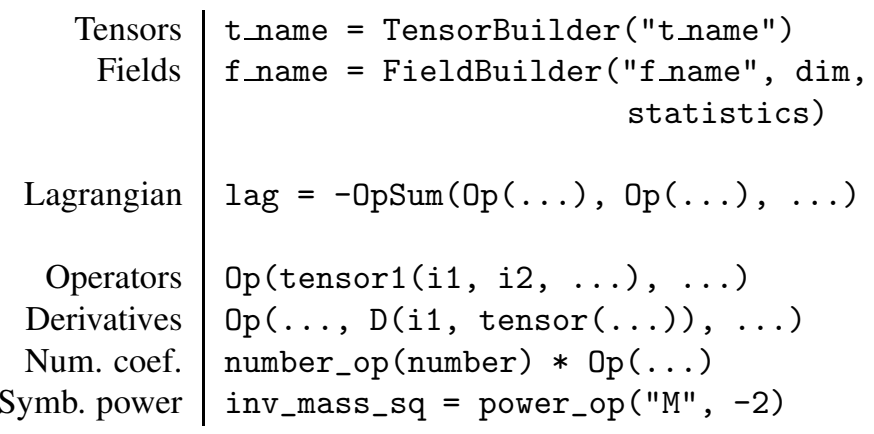

Table 1: Summary of the tools for the creation of a model.

- epsUp and epsDown: the totally anti-symmetric tensors $\epsilon^{\alpha \beta}$ and $\epsilon_{\alpha \beta}$ with two undotted two-component spinor indices defined by $\epsilon^{12}=-\epsilon^{21}=-\epsilon_{12}=\epsilon_{21}=1$.

- epsUpDot and epsDownDot: the totally anti-symmetric tensors $\epsilon^{\dot{\alpha} \dot{\beta}}$ and $\epsilon_{\dot{\alpha} \dot{\beta}}$ with two dotted two-component spinor indices given by $\epsilon_{\dot{\alpha} \dot{\beta}}=\left(\epsilon_{\alpha \beta}\right)^{*}$ and $\epsilon^{\dot{\alpha} \dot{\beta}}=\left(\epsilon^{\alpha \beta}\right)^{*}$.

- sigma4 and sigma4bar: the tensors $\sigma_{\alpha \dot{\alpha}}^{\mu}$ and $\bar{\sigma}_{\mu}^{\dot{\alpha} \alpha}$ given by $\sigma^{\mu}=\left(I_{2 \times 2}, \vec{\sigma}\right)$ and $\bar{\sigma}_{\mu}=\left(I_{2 \times 2},-\vec{\sigma}\right)$, where $\vec{\sigma}$ is the threevector of the Pauli matrices. The first index of sigma 4 and sigma4bar corresponds to the Lorentz index.

\section{Integration}

This section explains how to use the classes that represent the heavy fields as well as the function integrate, to integrate them out. They belong to the module matchingtools. integration. To import them do:

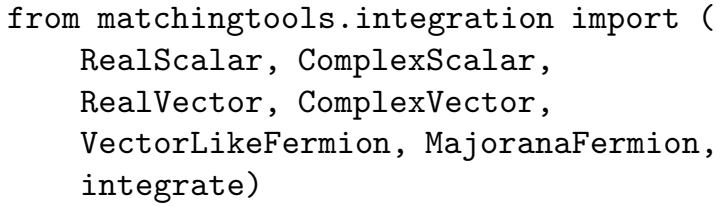

To integrate out the heavy fields from a previously defined Lagrangian we should specify which of the fields are heavy. This is done using the classes:

- RealScalar. Its constructor receives as arguments the name of the field and the number of indices it has.

- ComplexScalar. Requires a field-conjugate field pair. The arguments of the constructor are the name of the field, the name of its conjugate and its number of indices.

- RealVector. The arguments are the name of the field and the number of indices. The first index of the field is understood to be the Lorentz vector index.

- ComplexVector. The arguments are the name of the field, the name of its conjugate and the number of indices. The first index of both fields should be their corresponding Lorentz vector index. 
- VectorLikeFermion. The first argument of the constructor is the name of the field. The second and third are the names of the left-handed and right-handed parts. The fourth and fifth are their conjugates. The last is the number of indices. The first index of the each of the four fields is taken to be their two-component spinor index.

- MajoranaFermion. The arguments are the name of the field and the name of its conjugate. The first index of both fields should be their two-component spinor index.

The constructors for the bosons have the optional arguments: order (default 2), specifying the order in $(D / M)^{2}$ to which the solution to the equation of motion is to be expanded, and max_dim (default 4), representing the maximum allowed dimension for the operators appearing in this expansion. Both bosons and fermions receive the optional argument has_flavor (default True) stating whether the heavy field has a flavor index. In case it is true, the flavor index is taken to be the last one.

The heavy field classes include the quadratic terms for the kind of particle they represent, as well as the solutions to the equations of motion presented in section 2 The mass of a field $f$ is represented by a tensor whose name is of the form mass = "M" + f.name. This tensor has one index if the heavy field has flavor and none otherwise.

Therefore, the first step for integration is defining the heavy fields:

$$
\text { heavy_f }=\text { HeavyFieldClass ("field_name", ...) }
$$

Given an interaction Lagrangian int_lag, the integration is done using the function integrate, which takes as arguments a list of the heavy fields, the interaction Lagrangian and a maximum dimension max_dim for the operators of the effective theory. It returns the corresponding effective Lagrangian:

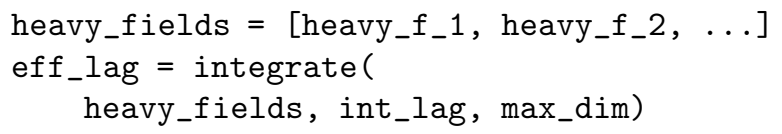

\section{Transformations of the effective Lagrangian}

After integration, the effective Lagrangian contains in general operators that are not independent. To rewrite it in terms of a set of independent operators some manipulations are needed, such as using identities for combinations of tensors related to the symmetry groups, integrating by parts to move derivatives from some fields to others, or using the equations of motion of the light fields.

The matchingtools.transformations module introduces the functions for doing this kind of manipulations and for the simplification of the Lagrangian. We will describe here the functions that are imported with

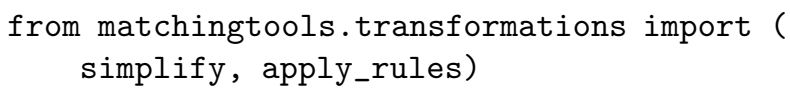

First, the function simplify returns a simplified version of the Lagrangian it gets as an argument. Tensors representing a number that appear inside an operator are collected and multiplied. Tensors representing a symbolic constant exponentiated to some power are also collected to give only one tensor with the correct exponent. simplify also looks for Kronecker deltas (tensors with the name "kdelta" and two indices) removes them by contracting the corresponding indices.

The transformations of a Lagrangian are done using what we call here rules. A rule is a pair (a tuple with two elements) whose first element is an operator representing a pattern and whose second element is an operator sum representing a replacement. They are used by the function apply_rules to find occurrences of the pattern and replace them by the replacement. A rule is written as

$$
\text { rule }=(O p(\ldots), O p \operatorname{Oum}(O p(\ldots), O p(\ldots), \ldots))
$$

The indices that appear in tensors inside the rule can be general integer numbers. Non-negative integers represent contracted indices, as explained in section 3 Negative indices are used for free indices and those in the replacement should match the corresponding ones in the pattern. For example the substitution of $\sigma_{i j}^{a} \sigma_{k l}^{b}$ by $2 \delta_{i l} \delta_{k j}-\delta_{i j} \delta_{k l}$ can be done using the rule

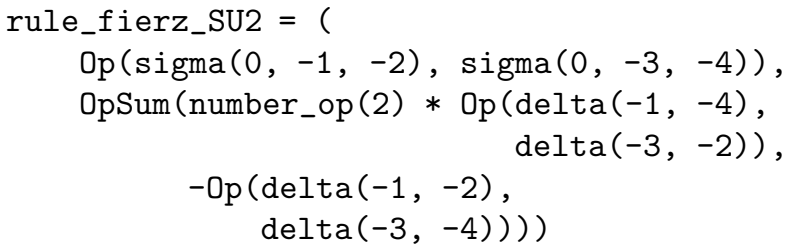

To transform the Lagrangian using integration by parts or equations of motion of the light fields the user should also specify the corresponding rules following this procedure.

The function apply_rules repeatedly tries to apply every rule of a list to each operator in an operator sum. If the pattern matches some part of an operator, the rule is applied and the operator sum updated. The first argument to apply_rules is the operator sum, the second is the list of rules and the last one is the number of iterations. It returns the resulting operator sum.

To rewrite the Lagrangian in terms of a chosen set of independent operators the procedure is: define the rules to get to the desired basis, add some rules to identify the operators and apply the function apply_rules.

The basis operators should be defined using tensor_op, a function that creates an operator with one tensor inside whose name is the argument of the function. Then write a rule to identify it. For example, for the operator $O_{\phi D}=\left(\phi^{\dagger} D_{\mu} \phi\right)\left(D^{\mu} \phi\right)^{\dagger} \phi$ we would write

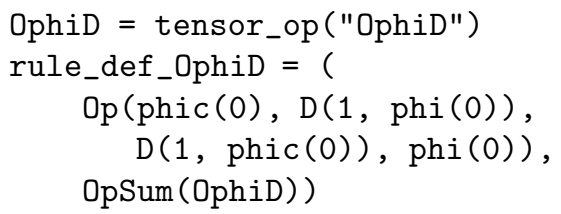


If the basis operator in question has some flavor indices, flavor_tensor_op is to be used instead of tensor_op. It creates a callable object that takes the corresponding free indices as arguments. As an example, for the operator $\left(O_{e \phi}\right)_{i j}=$ $\bar{l}_{L i} \phi e_{R j} \phi^{\dagger} \phi$ we would have:

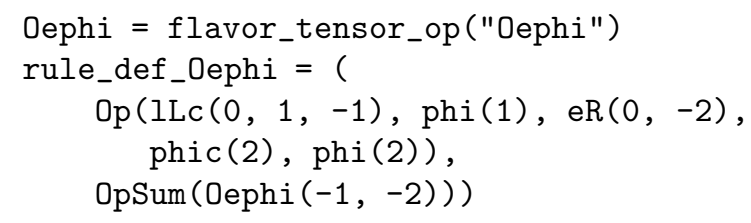

\section{Output}

The class matchingtools.output.Writer serves to nicely represent an effective Lagrangian. It is convenient that the final result is represented as a list of the coefficients of the operators in the basis. That is, if each of the terms of the Lagrangian contains a tensor that represents an operator of the basis, we would like to see what are the tensors that multiply each of them. This is what Writer does. If eff_lag is our final effective Lagrangian and op_names is a list of the names of the tensors representing the operators in the basis, do

$$
\text { eff_lag_writer = Writer(eff_lag, op_names) }
$$

The constructor admits an optional argument conjugates, a dictionary whose keys are the names of all the tensors involved in the final output and whose values are the names of their conjugates. This helps Writer collect pairs of conjugate products of tensors returning their real or imaginary part.

The string representation can be obtained just by using the str method of the class Writer. To write it to a text file use

$$
\text { eff_lag_writer.write_text_file(filename). }
$$

The method write_latex_file writes a LaTeX file with the representation. It receives four arguments: the name of the output file, the LaTeX representation of the tensors, the LaTeX representation of the coefficients of the basis operators and a list of the strings to be used to represent the indices. The LaTeX representations are given by dictionaries whose keys are the names of the tensors to be represented (or whose coefficient is to be represented) and whose values are the corresponding code. This code should contain placeholders for the necessary indices written as " \{\} " (Python's format style). To produce the characters " $\{$ ", " $\}$ " in the final code they should appear duplicated in the dictionary values.

For a better LaTeX output for the numerical coefficients, the parameter passed to number_op in the definitions should be either an int or a fractions.Fraction. In this context, the imaginary unit can be introduced by multiplying by the operator core.i_op.

\section{An example}

In this section we will be creating a simple model to show some of the features of MatchingTools. The model is described as follows: it has $S U(2) \times U(1)$ gauge symmetry and contains a complex scalar doublet $\phi$ (the Higgs) with hypercharge $1 / 2$ and a real scalar triplet $\Xi$ with zero hypercharge that couple as:

$$
\mathcal{L}_{\text {int }}=-\kappa \Xi^{a} \phi^{\dagger} \sigma^{a} \phi-\lambda \Xi^{a} \Xi^{a} \phi^{\dagger} \phi,
$$

where $\kappa$ and $\lambda$ are a coupling constants and $\sigma^{a}$ are the Pauli matrices. We will then integrate out the heavy scalar $\Xi$ to obtain an effective Lagrangian which we will finally write in terms of the operators

$$
\begin{aligned}
& O_{\phi 6}=\left(\phi^{\dagger} \phi\right)^{3}, \quad O_{\phi 4}=\left(\phi^{\dagger} \phi\right)^{2}, \\
& O_{\phi}^{(1)}=\phi^{\dagger} \phi\left(D_{\mu} \phi\right)^{\dagger} D^{\mu} \phi, \quad O_{\phi}^{(3)}=\left(\phi^{\dagger} D_{\mu} \phi\right)\left(D^{\mu} \phi\right)^{\dagger} \phi, \\
& O_{D \phi}=\phi^{\dagger}\left(D_{\mu} \phi\right) \phi^{\dagger} D^{\mu} \phi, \quad O_{D \phi}^{*}=\left(D_{\mu} \phi\right)^{\dagger} \phi\left(D^{\mu} \phi\right)^{\dagger} \phi .
\end{aligned}
$$

Notice that this is not an independent set of operators, as some linear combinations of them are total derivatives. Because the purpose of this section is to present a very simple model, we will not be doing integration by parts and therefore we will not simplify the results any further.

\subsection{Creation of the model}

The required imports are

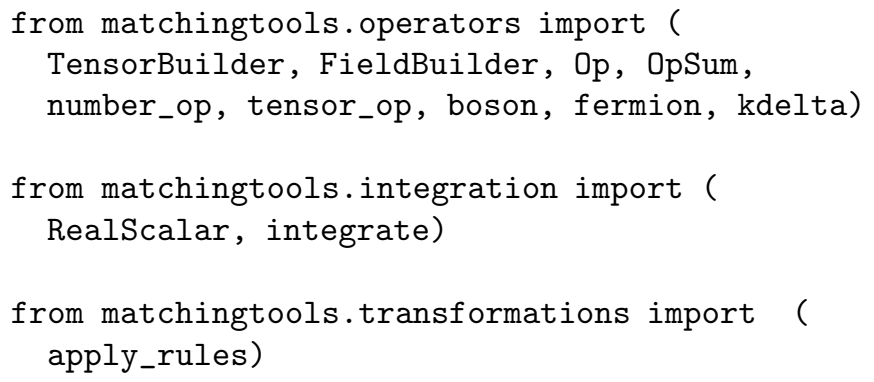

We will need three tensors, the Pauli matrices and the coupling constants:

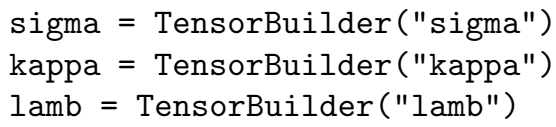

We will also use three fields: the Higgs doublet, its conjugate and the new scalar:

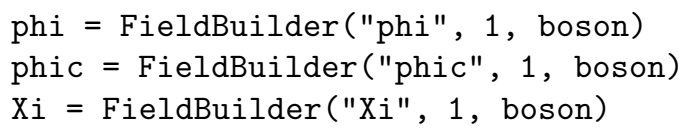

Now we are ready to write the interaction Lagrangian:

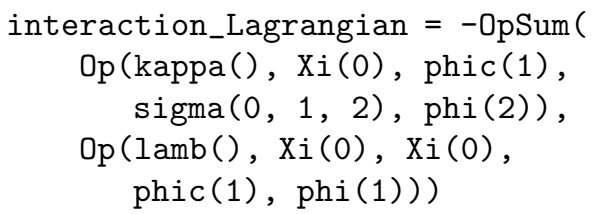




\subsection{Integration}

To integrate out the heavy $\Xi$ we write

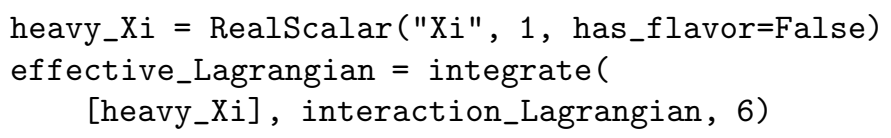

\subsection{Transformations of the effective Lagrangian}

After the integration we get operators that contain $\left(\phi^{\dagger} \sigma^{a} \phi\right)\left(\phi^{\dagger} \sigma^{a} \phi\right)$. This product can be rewritten in terms of the operator $\left(\phi^{\dagger} \phi\right)^{2}$. To do this, we can use the $S U(2)$ Fierz identity:

$$
\sigma_{i j}^{a} \sigma_{k l}^{a}=2 \delta_{i l} \delta_{k j}-\delta_{i j} \delta_{k l} .
$$

We now know that we can define a rule to transform everything that matches the left-hand side of the equality into the expression in the right-hand side with the code

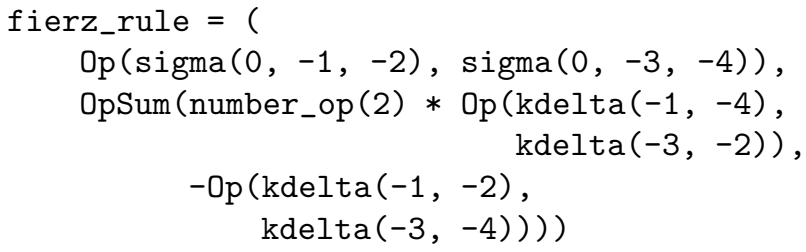

We should now define the operators in terms of which we want to express the effective Lagrangian

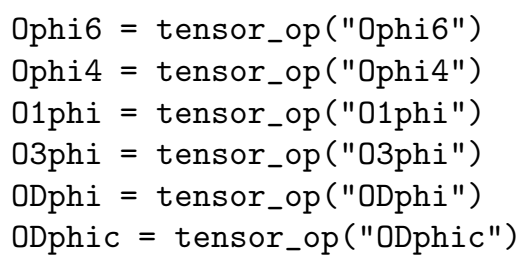

and then use some rules to express them in terms of the fields and tensors that appear in the effective Lagrangian

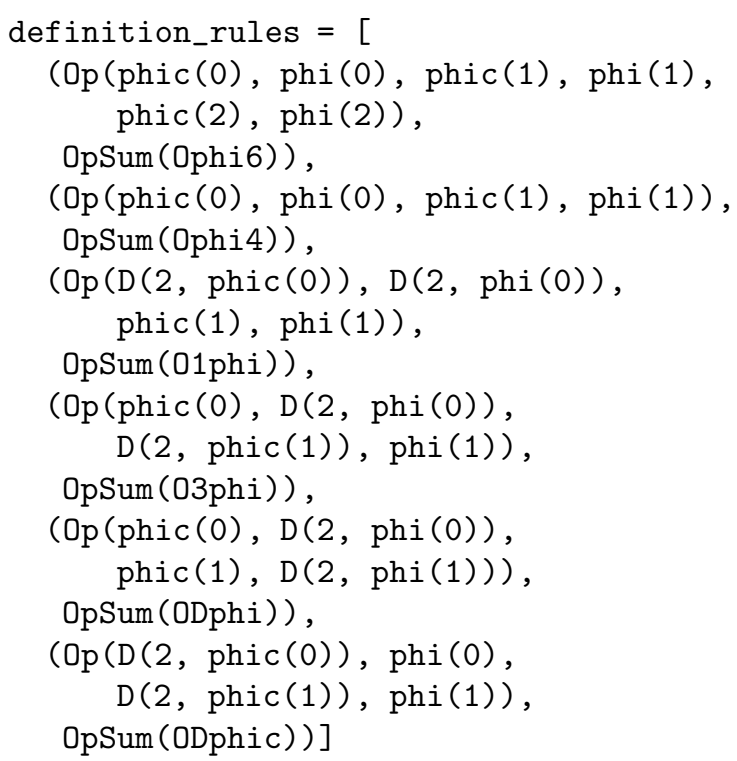

To apply the $S U(2)$ Fierz identity to every operator until we get to the chosen operators, we do

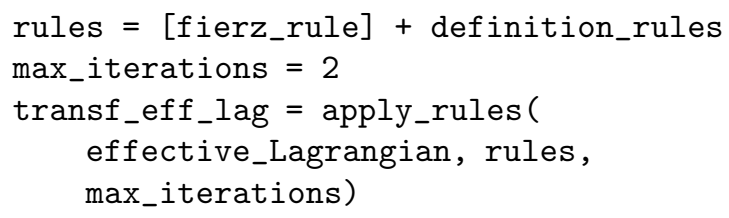

\subsection{Output}

The class Writer can be used to represent the coefficients of the operators of a Lagrangian as plain text and write them to a file

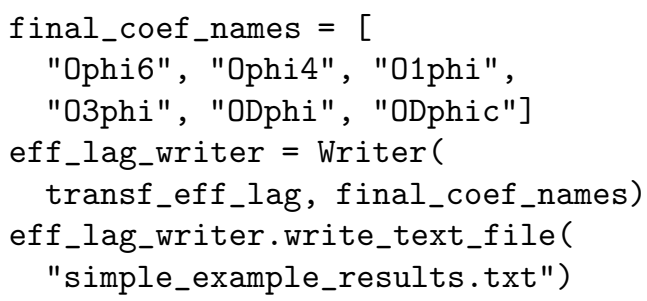

It can also write a LaTeX file with the representation of these coefficients and export it to pdf to show it directly. For this to be done, we should define how the objects that we are using are represented in LaTeX code and the symbols we want to be used as indices

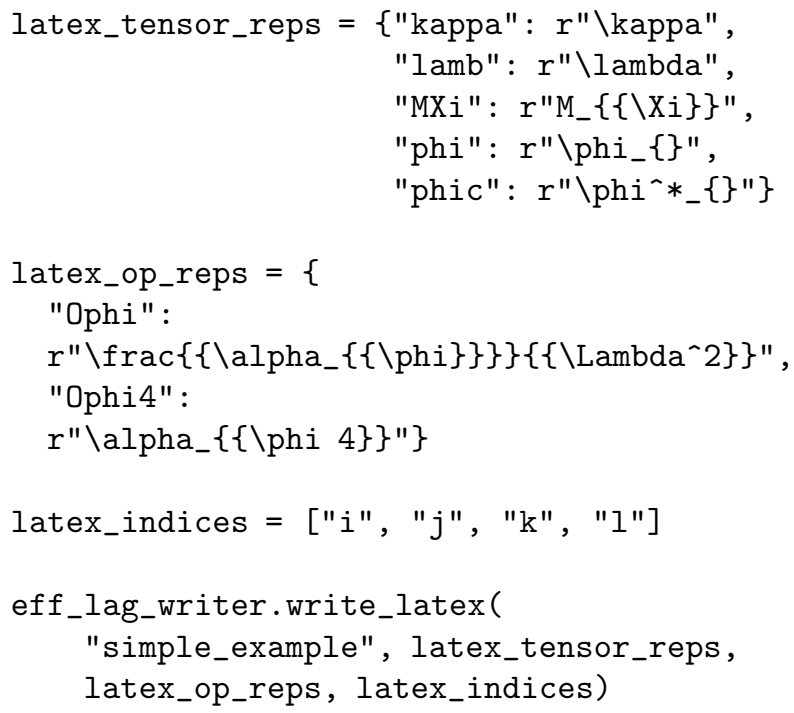

The expected result is a . tex file (ready to be compiled) with the coefficients of the operators we defined.

\section{Extras for beyond the Standard Model applications}

MatchingTools includes a subpackage called extras, with some modules defining tensors and rules that are useful for the applications to physics beyond the Standard Model. These modules are SU2, SU3, Lorentz, SM and SM_dim_6_basis. Other modules will be added in the future and will be available in the GitHub repository of the program, as well as in its updates in the pypi repository [55]. 


\subsection{The SU2 module}

This module defines the following tensors related to $S U(2)$ :

- epsSU2: The totally antisymmetric tensor $\epsilon_{i j}$ with two doublet indices and $\epsilon_{12}=1$.

- sigmaSU2: The Pauli matrices $\sigma_{i j}^{a}$. The first index is the triplet index, whereas the second and third are the doublet ones.

- CSU2 and CSU2c: the Clebsh-Gordan coefficients $C_{a \beta}^{I}$ with the first index $I$ being a quadruplet index, the second $a$ a triplet index, and the third $\beta$ a doublet index. The tensor $C$ contracted with the corresponding three objects produces a singlet.

- epsSU2triplets: Totally antisymmetric tensor $\epsilon_{a b c}$ with three $S U(2)$ triplet indices such that $\epsilon_{123}=1$.

- fSU2: Totally antisymmetric tensor with three $S U(2)$ triplet indices given by $f_{a b c}=\frac{i}{\sqrt{2}} \epsilon_{a b c}$.

It also implements the rules for taking expressions with $\epsilon_{i j} \epsilon_{k l}$, $\sigma_{i j}^{a} \sigma_{k l}^{a}, C_{a p}^{I} \epsilon_{p m} \sigma_{i j}^{a} C_{b q}^{I *} \epsilon_{q n} \sigma_{k l}^{b}$ or contractions of anti-symmetric tensors, and rewriting them in terms of Kronecker deltas. All the rules are collected in the list rules_SU2. The LaTeX representation of the tensors defined is given by the dictionary latex_SU2.

\subsection{The SU3 module}

The $S U(3)$ tensors defined in this module are:

- epsSU3: Totally antisymmetric tensor $\epsilon_{A B C}$ with three $S U(3)$ triplet indices such that $\epsilon_{123}=1$.

- TSU3: $S U(3)$ generators $\left(T_{A}\right)_{B C}=\frac{1}{2}\left(\lambda_{A}\right)_{B C}$, where $\lambda_{A}$ are the Gell-Mann matrices. The first index is the octet index. The second and third are the anti-triplet and triplet ones.

- fSU3: $S U(3)$ structure constants $f_{A B C}$.

The rule for transforming $\epsilon_{i j k} \epsilon_{i l m}$ into a combination of Kronecker deltas is implemented. It is included in the one-element list rules_SU3. The LaTeX representation of the tensors defined is in latex_SU3.

\subsection{The Lorentz module}

This module includes the tensors epsUp, epsUpDot, epsDown, epsDownDot, sigma4, sigma4bar from matchingtools. operators and defines:

- eps4: Totally antisymmetric tensor $\epsilon_{\mu \nu \rho \sigma}$ with four Lorentz vector indices where $\epsilon_{0123}=1$.

- sigmaTensor: Lorentz tensor

$$
\sigma^{\mu v}=\frac{i}{4}\left(\sigma_{\alpha \dot{\gamma}}^{\mu} \bar{\sigma}^{r \dot{\gamma} \beta}-\sigma_{\alpha \dot{\gamma}}^{v} \bar{\sigma}^{\mu \dot{\gamma} \beta}\right)
$$

The list rules_Lorentz contains the rules for substituting $\epsilon^{\alpha \beta} \epsilon^{\dot{\alpha} \dot{\beta}}$ by $\frac{1}{2} \bar{\sigma}^{\mu, \dot{\alpha} \alpha} \bar{\sigma}_{\mu}^{\dot{\beta} \beta}, \epsilon_{\alpha \beta} \epsilon_{\dot{\alpha} \dot{\beta}}$ by $\frac{1}{2} \bar{\sigma}_{\alpha \dot{\alpha}}^{\mu} \bar{\sigma}_{\mu, \beta \dot{\beta}}$ and contracted $\epsilon$ tensors by combinations of Kronecker deltas.

\subsection{The SM module}

Here, the tensors corresponding to the Standard Model fields and its gauge coupling constants, Yukawa couplings and CKM matrix are defined.

The Standard Model fields are:

- phi and phic: The Higgs boson and its conjugate. One $S U(2)$ doublet index.

- IL and ILc: The left-handed lepton doublet. Its indices are, in order: the two-component spinor index, the $S U(2)$ doublet index and the flavor index.

- qL and qLc: The left-handed quark doublet. Its indices are: the two-component spinor index, the $S U(3)$ triplet (or anti-triplet) index, the $S U(2)$ doublet index and the flavor index.

- eR and eRc: The right-handed electron. Indices: twocomponent spinor and flavor.

- $u R$ and uRc: The right-handed up quark. Indices: twocomponent spinor, $S U(3)$ triplet (or antitriplet) and flavor.

- $d R$ and dRc: The right-handed down quark. Indices: twocomponent spinor, $S U(3)$ triplet (or antitriplet) and flavor.

- bFS: $U(1)$ field strength tensor. Two Lorentz vector indices.

- wFS: $S U(2)$ field strength tensor. Two Lorentz vector indices and one $S U(2)$ triplet index.

- gFS: $S U(3)$ field strength tensor. Two Lorentz vector indices and one $S U(3)$ octet index.

The constant tensors are:

- gb and gw: The $U(1)$ and $S U(2)$ gauge coupling constants.

- ye, yec, yd, ydc, yu and yuc: The diagonalized Yukawa matrices for the leptons, the down quarks, the up quarks and their conjugates. They have two indices: the first one corresponds to the flavor of the doublets and the second to the flavor of the singlets.

- V and Vc: CKM matrix.

The module also includes a list of rules eoms_SM, defined to substitute the equations of motion, replacing derivatives of the Standard Model fields by a combination of the other fields. There is a dictionary latex_SM containing the LaTeX representation of the tensors that are defined.

\subsection{The SM_dim_6_basis module}

In this module, the basis for the Standard Model effective Lagrangian up to dimension six that appears in [29] is defined. The rules to identify them are given in the list rules_basis_definition. The LaTeX representation of their coefficients is in latex_basis_coefs. Modules containing other bases, such as the one in [46], will be added in the future. 


\section{Using MatchingTools with other types of fields}

As explained above, MatchingTools can integrate scalars, vector-like or Majorana fermions, and vectors in Lorentzinvariant theories. For this purpose, several classes representing the heavy fields are supplied. Other kinds of fields (for instance, with non canonical kinetic terms, spin $>1$, or non relativistic) can be treated as well, once the corresponding class for it is provided.

Specifically, to treat a new type of field one should define a Python class implementing the following methods:

- equations_of_motion. Receives an OperatorSum object representing an interaction Lagrangian. Returns a dictionary whose keys are strings with the names of the heavy fields involved (for example, a field and its conjugate, if it is a complex boson) and whose values are OperatorSum objects representing the corresponding solution to their equation of motion. These solutions can be written in terms of other heavy fields, but they should be such that iterative substitutions of their respective equations motion reaches a point where no heavy fields appear to the desired order in the dimension of the operators.

- quadratic_terms. Does not have any parameters. Returns the kinetic and mass terms of the corresponding heavy field.

For the definition of these methods, it is recommended to use the tools provided by the core module. Once such a class is defined, its objects can be included in the list of heavy fields to be passed to integration. integrate and they will be dealt with in the same way as the others.

\section{Conclusions}

We have presented MatchingTools, a Python library implementing symbolic tree-level integration of heavy fields for any given model. It is also able to transform the resulting Lagrangian using rules specified by the user to remove redundant operators. With this program one can safely automatize these kind of calculations, which practically eliminates the possibility of algebraic errors and drastically reduces the calculation times. Even calculations with complex Lagrangians involving $\sim 100$ independent terms (thousands of terms in some intermediate steps) can be performed in about thirty seconds (using a 2.6 GHz Intel Core i5 processor).

\section{Acknowledgments}

The author would like to thank J. de Blas, M. Pérez-Victoria and J. Santiago for their very useful guidance, comments and corrections.

Funding: This work was supported by the Spanish MECD grant FPU14, the Spanish MINECO grants FPA2013-47836C3-2-P and FPA2016-78220-C3-1-P (Fondos FEDER) and the Junta de Andalucía grant FQM101.

\section{References}

[1] H. Georgi, Weak Interactions and Modern Particle Theory, 1984.

[2] J. F. Donoghue, E. Golowich, B. R. Holstein, Dynamics of the standard model, Camb. Monogr. Part. Phys. Nucl. Phys. Cosmol. 2 (1992) 1-540, [Camb. Monogr. Part. Phys. Nucl. Phys. Cosmol.35(2014)].

[3] M. Aaboud, et al., Search for scalar leptoquarks in pp collisions at $\sqrt{s}=$ $13 \mathrm{TeV}$ with the ATLAS experiment, New J. Phys. 18 (9) (2016) 093016. arXiv:1605.06035 doi:10.1088/1367-2630/18/9/093016

[4] M. Aaboud, et al., Search for pair production of vector-like top quarks in events with one lepton, jets, and missing transverse momentum in $\sqrt{s}=$ $13 \mathrm{TeV} p p$ collisions with the ATLAS detector, JHEP 08 (2017) 052. arXiv:1705.10751 doi:10.1007/JHEP08(2017) 052

[5] A. M. Sirunyan, et al., Searches for W bosons decaying to a top quark and a bottom quark in proton-proton collisions at $13 \mathrm{TeV}$, JHEP 08 (2017) 029. arXiv: 1706.04260 doi:10.1007/JHEP08(2017) 029

[6] A. M. Sirunyan, et al., Search for heavy resonances that decay into a vector boson and a Higgs boson in hadronic final states at $\sqrt{\mathrm{s}}=$ 13 TeV, Eur. Phys. J. C77 (9) (2017) 636. arXiv:1707.01303 doi:10.1140/epjc/s10052-017-5192-z

[7] I. Brivio, M. Trott, The Standard Model as an Effective Field Theory, arXiv:1706.08945

[8] Z. Han, W. Skiba, Effective theory analysis of precision electroweak data, Phys. Rev. D71 (2005) 075009. arXiv:hep-ph/0412166 doi:10.1103/PhysRevD.71.075009

[9] J. de Blas, M. Chala, J. Santiago, Global Constraints on LeptonQuark Contact Interactions, Phys. Rev. D88 (2013) 095011. arXiv:1307.5068 doi:10.1103/PhysRevD.88.095011

[10] J. de Blas, Electroweak limits on physics beyond the Standard Model, EPJ Web Conf. 60 (2013) 19008. arXiv:1307.6173 doi:10.1051/epjconf/20136019008

[11] R. S. Gupta, A. Pomarol, F. Riva, BSM Primary Effects, Phys. Rev. D91 (3) (2015) 035001. arXiv:1405.0181 doi:10.1103/PhysRevD.91.035001

[12] J. Ellis, V. Sanz, T. You, The Effective Standard Model after LHC Run I, JHEP 03 (2015) 157. arXiv:1410.7703 doi:10.1007/JHEP03(2015) 157

[13] A. Falkowski, F. Riva, Model-independent precision constraints on dimension-6 operators, JHEP 02 (2015) 039. arXiv:1411.0669 doi: 10.1007/JHEP02(2015) 039

[14] J. de Blas, M. Chala, J. Santiago, Renormalization Group Constraints on New Top Interactions from Electroweak Precision Data, JHEP 09 (2015) 189. arXiv:1507.00757 doi:10.1007/JHEP09(2015)189

[15] L. Berthier, M. Trott, Consistent constraints on the Standard Model Effective Field Theory, JHEP 02 (2016) 069. arXiv:1508.05060 doi:10.1007/JHEP02(2016) 069

[16] A. Pomarol, F. Riva, Towards the Ultimate SM Fit to Close in on Higgs Physics, JHEP 01 (2014) 151. arXiv:1308.2803 doi:10.1007/JHEP01(2014)151

[17] J. de Blas, M. Ciuchini, E. Franco, S. Mishima, M. Pierini, L. Reina, L. Silvestrini, The Global Electroweak and Higgs Fits in the LHC era, 2017. arXiv: 1710.05402

[18] T. Appelquist, C. W. Bernard, Strongly Interacting Higgs Bosons, Phys. Rev. D22 (1980) 200. doi:10.1103/PhysRevD.22.200

[19] A. C. Longhitano, Heavy Higgs Bosons in the Weinberg-Salam Model, Phys. Rev. D22 (1980) 1166. doi:10.1103/PhysRevD.22.1166

[20] A. C. Longhitano, Low-Energy Impact of a Heavy Higgs Boson Sector, Nucl. Phys. B188 (1981) 118-154. doi:10.1016/0550-3213(81)90109-7

[21] F. Feruglio, The Chiral approach to the electroweak interactions, Int. J. Mod. Phys. A8 (1993) 4937-4972. arXiv:hep-ph/9301281 doi:10.1142/S0217751X93001946

[22] H. Georgi, S. L. Glashow, Unity of All Elementary Particle Forces, Phys. Rev. Lett. 32 (1974) 438-441. doi:10.1103/PhysRevLett.32.438

[23] H. Fritzsch, P. Minkowski, Unified Interactions of Leptons and Hadrons, Annals Phys. 93 (1975) 193-266. doi:10.1016/0003-4916(75)90211-0

[24] N. Arkani-Hamed, S. Dimopoulos, G. R. Dvali, The Hierarchy problem and new dimensions at a millimeter, Phys. Lett. B429 (1998) 263-272. arXiv:hep-ph/9803315 doi:10.1016/S0370-2693(98)00466-3

[25] L. Randall, R. Sundrum, A Large mass hierarchy from a 
small extra dimension, Phys, Rev. Lett. 83 (1999) 3370-3373. arXiv:hep-ph/9905221 doi:10.1103/PhysRevLett.83.3370

[26] F. del Aguila, M. Perez-Victoria, J. Santiago, Observable contributions of new exotic quarks to quark mixing, JHEP 09 (2000) 011 arXiv:hep-ph/0007316 doi:10.1088/1126-6708/2000/09/011

[27] F. del Aguila, J. de Blas, M. Perez-Victoria, Effects of new leptons in Electroweak Precision Data, Phys. Rev. D78 (2008) 013010 arXiv:0803.4008 doi:10.1103/PhysRevD.78.013010

[28] F. del Aguila, J. de Blas, M. Perez-Victoria, Electroweak Limits on General New Vector Bosons, JHEP 09 (2010) 033. arXiv:1005.3998 doi:10.1007/JHEP09(2010)033

[29] J. de Blas, M. Chala, M. Perez-Victoria, J. Santiago, Observable Effects of General New Scalar Particles, JHEP 04 (2015) 078. arXiv: 1412.8480 doi:10.1007/JHEP04(2015) 078

[30] C. M. Fraser, Calculation of Higher Derivative Terms in the One Loop Effective Lagrangian, Z. Phys. C28 (1985) 101. doi:10.1007/BF01550255

[31] I. J. R. Aitchison, C. M. Fraser, Fermion Loop Contribution to Skyrmion Stability, Phys. Lett. 146B (1984) 63-66. doi:10.1016/0370-2693(84)90644-0

[32] I. J. R. Aitchison, C. M. Fraser, Derivative Expansions of Fermion Determinants: Anomaly Induced Vertices, Goldstone-Wilczek Currents and Skyrme Terms, Phys. Rev. D31 (1985) 2605. doi:10.1103/PhysRevD.31.2605

[33] I. J. R. Aitchison, C. M. Fraser, Trouble With Boson Loops in Skyrmion Physics, Phys. Rev. D32 (1985) 2190. doi:10.1103/PhysRevD.32.2190

[34] L. H. Chan, EFFECTIVE ACTION EXPANSION IN PERTURBATION THEORY, Phys. Rev. Lett. 54 (1985) 1222-1225, [Erratum: Phys. Rev. Lett.56,404(1986)]. doi:10.1103/PhysRevLett.54.1222

[35] L.-H. Chan, Derivative Expansion for the One Loop Effective Actions With Internal Symmetry, Phys. Rev. Lett. 57 (1986) 1199. doi:10.1103/PhysRevLett.57.1199

[36] M. K. Gaillard, The Effective One Loop Lagrangian With Derivative Couplings, Nucl. Phys. B268 (1986) 669-692. doi:10.1016/0550-3213(86)90264-6

[37] O. Cheyette, Derivative Expansion of the Effective Action, Phys. Rev. Lett. 55 (1985) 2394. doi : 10.1103/PhysRevLett.55. 2394

[38] F. del Aguila, Z. Kunszt, J. Santiago, One-loop effective lagrangians after matching, Eur. Phys. J. C76 (5) (2016) 244. arXiv:1602.00126 doi:10.1140/epjc/s10052-016-4081-1

[39] S. A. R. Ellis, J. Quevillon, T. You, Z. Zhang, Mixed heavylight matching in the Universal One-Loop Effective Action, Phys. Lett. B762 (2016) 166-176. arXiv:1604.02445 doi:10.1016/j.physletb.2016.09.016

[40] J. Fuentes-Martin, J. Portoles, P. Ruiz-Femenia, Integrating out heavy particles with functional methods: a simplified framework, JHEP 09 (2016) 156. arXiv:1607.02142 doi:10.1007/JHEP09(2016)156

[41] Z. Zhang, Covariant diagrams for one-loop matching, JHEP 05 (2017) 152. arXiv: 1610.00710 doi:10.1007/JHEP05(2017)152

[42] R. E. Kallosh, I. V. Tyutin, The Equivalence theorem and gauge invariance in renormalizable theories, Yad. Fiz. 17 (1973) 190-209, [Sov. J. Nucl. Phys.17,98(1973)].

[43] H. D. Politzer, Power Corrections at Short Distances, Nucl. Phys. B172 (1980) 349-382. doi:10.1016/0550-3213(80)90172-8

[44] H. Georgi, On-shell effective field theory, Nucl. Phys. B361 (1991) 339 350. doi:10.1016/0550-3213(91) 90244-R

[45] C. Arzt, Reduced effective Lagrangians, Phys. Lett B342 (1995) 189-195. arXiv:hep-ph/9304230 doi:10.1016/0370-2693(94)01419-D

[46] B. Grzadkowski, M. Iskrzynski, M. Misiak, J. Rosiek, DimensionSix Terms in the Standard Model Lagrangian, JHEP 10 (2010) 085 arXiv: 1008.4884 doi:10.1007/JHEP10(2010)085

[47] R. Contino, M. Ghezzi, C. Grojean, M. Muhlleitner, M. Spira, Effective Lagrangian for a light Higgs-like scalar, JHEP 07 (2013) 035. arXiv:1303.3876 doi:10.1007/JHEP07(2013)035

[48] E. Masso, An Effective Guide to Beyond the Standard Model Physics, JHEP 10 (2014) 128. arXiv:1406.6376 doi:10.1007/JHEP10(2014)128

[49] A. Falkowski, B. Fuks, K. Mawatari, K. Mimasu, F. Riva, V. sanz, Rosetta: an operator basis translator for Standard Model effective field theory, Eur. Phys. J. C75 (12) (2015) 583. arXiv:1508.05895 doi:10.1140/epjc/s10052-015-3806-x

[50] J. de Blas, J. C. Criado, M. Perez-Victoria, J. Santiago, Effective description of general extensions of the Standard Model: the complete tree-level dictionary, JHEP 03 (2018) 109. arXiv:1711.10391 doi:10.1007/JHEP03(2018) 109

[51] N. D. Christensen, C. Duhr, FeynRules - Feynman rules made easy, Comput. Phys. Commun. 180 (2009) 1614-1641. arXiv:0806.4194 doi:10.1016/j.cpc.2009.02.018

[52] A. Alloul, N. D. Christensen, C. Degrande, C. Duhr, B. Fuks, FeynRules 2.0 - A complete toolbox for tree-level phenomenology, Comput. Phys. Commun. 185 (2014) 2250-2300. arXiv:1310.1921 doi:10.1016/j.cpc.2014.04.012

[53] A. Celis, J. Fuentes-Martin, A. Vicente, J. Virto, DsixTools: The Standard Model Effective Field Theory Toolkit, Eur. Phys. J. C77 (6) (2017) 405. arXiv:1704.04504 doi:10.1140/epjc/s10052-017-4967-6

[54] I. Brivio, Y. Jiang, M. Trott, The SMEFTsim package, theory and tools arXiv: 1709.06492

[55] pip: The pypa recommended tool for installing python packages URL https://pypi.python .org/pypi/pip/

[56] H. K. Dreiner, H. E. Haber, S. P. Martin, Two-component spinor techniques and Feynman rules for quantum field theory and supersymmetry, Phys. Rept. 494 (2010) 1-196. arXiv:0812.1594 doi:10.1016/j.physrep.2010.05.002 